\title{
Case 2 - Trabectedin as a maintenance therapy in a patient with recurrent ovarian cancer and BRCA1 germline mutation
}

\author{
Filippo Greco ${ }^{1}$, Paolo Piacentini ${ }^{1}$, Andrea Remo ${ }^{2}$, Andrea Bonetti ${ }^{1}$ \\ Commentary: Gabriella Ferrandina
}

\begin{abstract}
Treatment of patients with recurrent ovarian cancer (ROC) is complex. This case report describes the effects of a sequence of treatments in a BRCA1 mutation carrier with serous papillary poorly differentiated, platinumsensitive ROC. This consisted in primary surgery, adjuvant chemotherapy, secondary cytoreduction with Heated IntraPEritoneal Chemotherapy (HIPEC), 12 cycles of trabectedin in combination with pegylated liposomal doxorubicin (PLD) and 12 cycles of trabectedin alone, leading to complete radiological and biochemical response.
\end{abstract}

Key words: BRCA, recurrent ovarian cancer, trabectedin

\section{Introduction}

Approximately $80 \%$ of patients with ovarian cancer will relapse (median progression-free survival [PFS] 18 months) and require second and further lines of therapy. Treatment of these patients is complex and several factors need to be considered, including the length of the platinum-free interval (PFI) [1].

Stratification of recurrent ovarian cancer (ROC) patients into different subgroups by PFI ( 4 weeks to $<6$ months, 6-12 months, and $>12$ months) is useful to define specific treatments within the 6- to 12 -month period for patients with partially platinum sensitive disease. In this clinical setting, the potential benefit of artificially extending the PFI has been evaluated in several studies, with data suggesting that this approach may restore platinum sensitivity and lead to clinical responses or stable disease with platinum retreatment, even in heavily pre-treated patients [2]. Trabectedin, a marine derived anti-neoplastic agent, has been shown to have clinical benefit in patients with ROC [3]. Trabectedin was initially isolated from the

'Oncology Unit, Oncology Department,

ULSS 21 Regione Veneto, Legnago (Verona), Italy.

2Pathology Unit, ULSS 21 Regione Veneto,

Legnago (Verona), Italy.

Correspondence to: Filippo Greco MD,

UOC di Oncologia, Ospedale Mater Salutis,

ULSS 21 Regione Veneto, 37045 Legnago (Verona), Italy.

Phone: +39 0442622364 - Fax: +390442622469

E-mail: filippo.greco@aulsslegnago.it

CANCER BREAKING NEWS 2016;4(1):41-44

DOI: $10.19156 / \mathrm{cbn} .2016 .0008$ tunicate Ecteinascidia turbinata and is currently produced synthetically. It was first approved in the European Union in 2007 as monotherapy for the treatment of patients with advanced soft tissue sarcoma (STS). In patients with relapsed, platinum-sensitive ovarian cancer, trabectedin was approved in 2009 for use in combination with pegylated liposomal doxorubicin (PLD). Early phase II trials showed that trabectedin had encouraging activity as a single agent in patients with relapsed ovarian cancer, particularly with platinum-sensitive disease [4]. A large, multicenter, randomized phase III trial (OVA-301) confirmed that trabectedin + PLD improved PFS and overall survival (OS) compared with PLD alone in the 6- to 12-month PFI subgroup [5].

This case report highlights an interesting sequencing of treatments in a BRCA1 mutation carrier who achieved a complete response during treatment with PLD and trabectedin, and subsequent trabectedin monotherapy.

\section{Case report}

In August 2011, a 49-year-old woman with a family history of breast cancer underwent hysterectomy with bilateral salpingo-oophorectomy, omentectomy, pelvic and lomboaortic linfadenectomy for ovarian serous papillary carcinoma (FIGO stage IIIC); pre-surgery CA125 level was $1388 \mathrm{U} / \mathrm{mL}$. The patient then started adjuvant chemotherapy with weekly paclitaxel $80 \mathrm{mg} / \mathrm{m}^{2}$ and carboplatin AUC5 every 3 weeks. After 2 cycles this schedule was discontinued due to grade 3 thrombocytopenia and grade 4 neutropenia. The patient continued with paclitaxel $175 \mathrm{mg} / \mathrm{m}^{2}$ every 
3 weeks and carboplatin AUC5; after 2 cycles the CA125 level had fallen to $25 \mathrm{U} / \mathrm{mL}$. Adjuvant chemotherapy ended in January 2012 and follow-up consisted of 4-monthly follow-up visits that included full-body computed tomographic (CT) scan and determination of CA125 levels. Over this period the patient received the results of an informative BRCA1 mutation test, which was positive. In June 2013 there was an increase in the CA125 serum level $(133 \mathrm{U} / \mathrm{mL})$ but full-body CT and 18FdG-PET scans showed no evidence of disease. Two months later, the CA125 level was $93 \mathrm{U} / \mathrm{mL}$, but after another 2 months had increased dramatically to $490 \mathrm{U} / \mathrm{mL}$; explorative laparoscopy showed evidence of massive peritoneal carcinosis. The patient underwent open complete pelvic peritonectomy, sigmoid colon resection, omentectomy, diaphragmatic peritonectomy, resection of multiple peritoneal nodules, and vaporization of nodules $<1 \mathrm{~mm}$, and there was no residual macroscopical disease. After surgery, Heated IntraPEritoneal Chemotherapy (HIPEC) with cisplatin $100 \mathrm{mg} / \mathrm{m}^{2}$ $\left(41^{\circ} \mathrm{C}\right)$ was performed over 60 minutes using the closed abdomen technique.

In June 2014, 8 months after the administration of intraperitoneal cisplatin, the serum CA125 level was $1123 \mathrm{U} / \mathrm{mL}$ and a $18 \mathrm{FdG}-\mathrm{PET}$ scan showed progressive peritoneal disease (PD) (Figure 1). The patient's Eastern Cooperative Oncology Group (ECOG) performance status was 0 , so a decision was made to start chemotherapy with PLD $\left(30 \mathrm{mg} / \mathrm{m}^{2}\right)$ and trabectedin $\left(1.1 \mathrm{mg} / \mathrm{m}^{2}\right)$ administered every 21 days. A total of 12 cycles were administered, and complete radiological response was achieved after just 3 cycles (Figure 2); the CA125 level decreased slowly and was $54 \mathrm{U} / \mathrm{mL}$ at the 12 th chemotherapy cycle. The patient experienced grade 3 hand-foot erythrodysesthesia and requested that treatment be stopped. It was suggested that she continue therapy with monthly trabectedin alone $\left(1.1 \mathrm{mg} / \mathrm{m}^{2}\right)$ as maintenance. The patient agreed and the CA125 level reached the normal range at the 14th cycle (second maintenance cycle). In February 2016, the patient received the 24th cycle of therapy (12 maintenance cycles) and continued to show complete radiological and biochemical response.

\section{Conclusion}

Despite a relatively poor long-term prognosis for patients diagnosed with ovarian cancer, the tide is beginning to turn towards optimizing treatment options for those with advanced recurrent disease. In contrast with first-line therapy where there is an excellent correlation between PFS and OS, this correlation is less clear for recurrent disease. Stratification of ROC patients according to the PFI is helpful to individualize therapeutic ap-

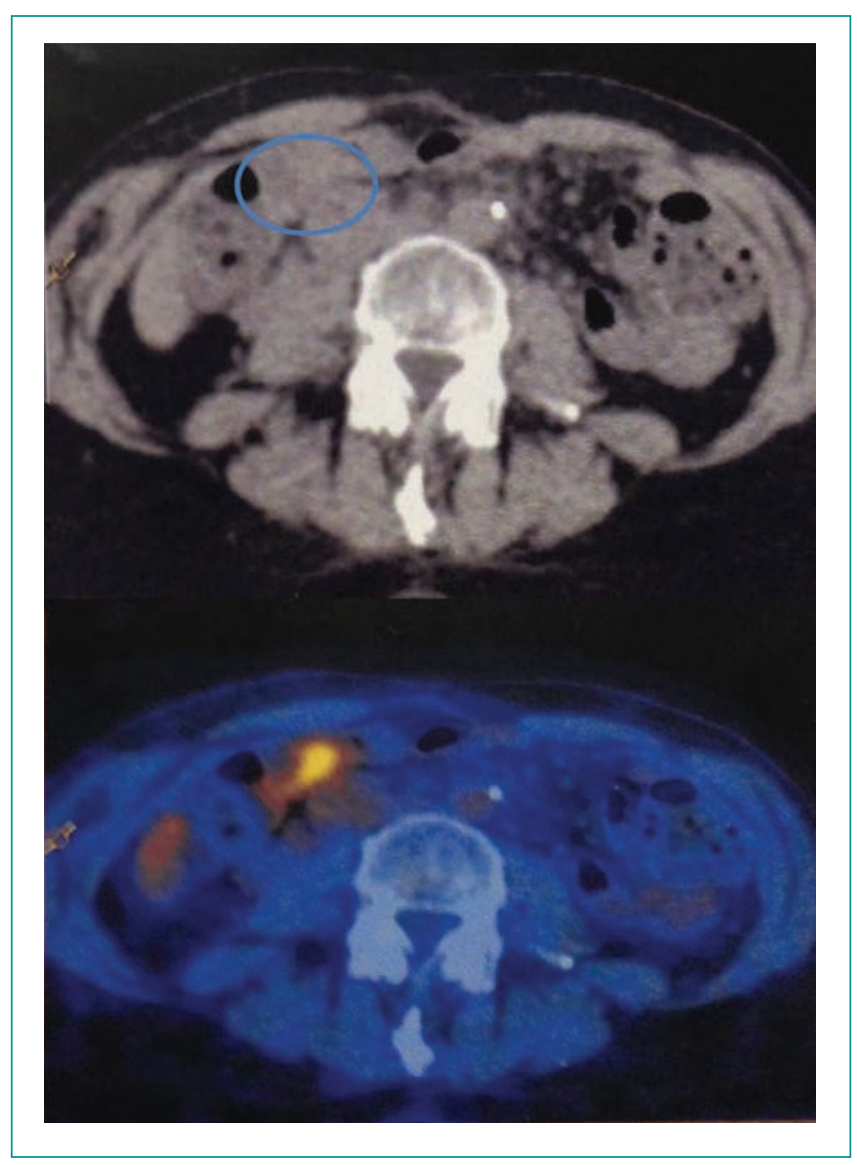

Fig. 1. Detection of progressive peritoneal disease with $18 \mathrm{FdG}-$ PET and CT scans.



Fig. 2. Achievement of complete radiological response after just 3 cycles with pegylated liposomal doxorubicin + trabectedin.

proaches for the different subgroups, and in particular to manage those with partially platinum-sensitive (PPS) disease. There is growing evidence for a survival benefit with non-platinum treatment regimens, such as the trabectedin + PLD combination, in patients with PPS disease when these patients are subsequently rechallenged with platinum. In the OVA-301 trial there was no statistically significant OS difference in the platinum-sensitive group (PFI $\geq 6$ months) [5]; median OS was 27.0 months 
in the trabectedin + PLD arm and 24.1 months in the PLD monotherapy arm. However, there was some suggestion of an OS difference in the 6-12 month PFI subgroup; median OS was 22.4 months in the trabectedin + PLD arm compared with 16.4 months in the PLD monotherapy arm [5]. In addition, the subgroup of patients with the BRCA1 mutation had a significantly longer median PFS than those without this mutation (13.6 versus 5.5 months, respectively) [5].

Ovarian cancer patients who respond to platinum-based chemotherapy have a reasonable likelihood of responding to a second or third re-introduction of a platinum-based regimen. It is also known that the statistical likelihood of an individual ovarian cancer patient experiencing clinicallyrelevant benefit from retreatment with a platinum regimen is a continuum, with increasing response rates associated with longer platinum-free periods. In the PPS subgroup, administration of a combination of trabectedin + PLD artificially prolongs the PFI, and a longer PFI predicts longer survival. In patients who respond to the combination of trabectedin and PLD, another approach to prolonging the PFI may be the administration of maintenance therapy with trabectedin alone. The prospective, phase 2, MITO-15 trial demonstrated the value of trabectedin monotherapy in patients with platinum-sensitive advanced ovarian cancer with BRCA mutation following failed treatment with multiple platinum regimens [6]. In that study, 94 patients were evaluable for response in the platinum-resistant and -sensitive populations, with overall response rates (ORR) of $31.2 \%$ and $47.8 \%$, respectively, and corresponding overall clinical benefit rates of $54.2 \%$ and $73.9 \%$. In 69 patients with recurrent BRCA-mutated ovarian cancer, there was no difference in ORR, PFS and OS according to BRCA 1-2 status.

The authors concluded that repeated platinum sensitivity identifies patients highly responsive to trabectedin, and that the activity of this agent appears comparable to that of platinum compounds in these patients [6].

\section{Acknowledgments}

The authors thank Nicola Ryan, an independent medical writer, who provided native English editing and journal styling on behalf of HPS. This editorial assistance was funded by PharmaMar, Spain.

\section{Conflicts of Interest}

The Authors declare there are no conflicts of interest in relation to this article.

\section{References}

1. Colombo N. Optimizing treatment of the partially platinum-sensitive ovarian cancer patient. Future Oncol 2013;9 (12s):19-23.

2. Kaye S. Management of partially platinum-sensitive relapsed ovarian cancer. Eur J Cancer 2008;6(Suppl):16-21.

3. Carter NJ, Keam SJ. Trabectedin: A review of its use in the management of soft tissue sarcoma and ovarian cancer. Drugs 2007;67:2257-76.

4. Krasner CN, McMeekin DS, Chan S, et al. A phase II study of trabectedin single agent in patients with recurrent ovari-

an cancer previously treated with platinum-based regimens. Br J Cancer 2007;97:1618-24.

5. Monk BJ, Herzog TJ, Kaye SB, et al. Trabectedin plus pegylated liposomal doxorubicin in recurrent ovarian cancer. J Clin Oncol 2010;28:3107-14.

6. Lorusso D, Scambia G, Pignata S, et al. Prospective phase II trial of trabectedin in BRCA-mutated and/or BRCAness phenotype recurrent ovarian cancer patients: the MITO 15 trial. Ann Oncol 2016 Mar;27(3):487-93.

\section{Commentary}

The present case highlights an emerging issue in gynecologic oncology, represented by the need to provide better rationale for the optimal sequence of drugs in the treatment of relapsed ovarian cancer patients. Indeed, based on the evidence from the Cancer Genome Atlas project [1] and present indepth understanding about the role of the BRCA mutation and BRCA phenotypes in ovarian cancer management [2], the optimal use of drugs in a logical, coordinated sequence for this poor prognosis disease is becoming more and more important.

Based on its unique mechanism of action, trabectedin has been shown to be particularly effective in cells lacking functional homologous recombinant mechanisms, such as those that have the BRCA mutation [3]. This provides a rational explanation for some clinical observations, with trabectedin showing good efficacy in sarcoma or breast cancer patients with the BRCA mutation [4, 5]. Moreover, recent observations in the OVA-301 study have underlined the superiority of the trabectedin + pegylated liposomal doxorubicin (PLD) combination versus PLD alone in recurrent ovarian cancer 
patients with the BRCA mutation [6]. Also, data from the recently published MITO-15 phase II study suggest that trabectedin monotherapy may be beneficial for ROC patients endowed with the BRCA mutation or BRCA-ness phenotype [7]. In this setting, it is important to take into account the demonstrated efficacy of trabectedin + PLD in artificially prolonging the duration of platinum-free interval, thus increasing the likelihood of response to subsequent platinum re-challenge [8]. Indeed, a higher responsiveness to platinum-containing regimens is also reasonably expected to result in an increased chance of taking advantage of the PARP inhibitors [9], thus hopefully leading to the best use of each $\operatorname{drug}(s)$ in the context of each patient's clinical history and biomolecular profile.

Gabriella Ferrandina

Gynecologic Oncology Unit, Catholic University, Rome, Italy E-mail: gabriella.ferrandina@rm.unicatt.it

\section{References}

1. Cancer Genome Atlas Research Network. Integrated genomic analyses of ovarian carcinoma. Nature 2011;474(7353): 609-15.

2. Pothuri B. BRCA1- and BRCA2-related mutations: therapeutic implications in ovarian cancer. Ann Oncol 2013;24(Suppl 8):viii22-7.

3. D'Incalci M, Galmarini CM. A review of trabectedin (ET-743): a unique mechanism of action. Mol Cancer Ther 2010;9(8):2157-63.

4. Erba E, Bergamaschi D, Bassano L, et al. Ecteinascidin-743 (ET-743), a natural marine compound, with a unique mechanism of action. Eur J Cancer 2001;37(1):97-105.

5. Delaloge S, Wolp-Diniz R, Byrski T, et al. Activity of trabectedin in germline BRCA1/2-mutated metastatic breast cancer: results of an international first-in-class phase II study. Ann Oncol 2014;25(6):1152-8.

6. Monk BJ, Ghatage P, Parekh T, et al. Effect of BRCA1 and XPG mutations on treatment response to trabectedin and pegylated liposomal doxorubicin in patients with advanced ovarian cancer: exploratory analysis of the phase 3 OVA301 study. Ann Oncol 2015 May;26(5):914-20.

7. Lorusso D, Scambia G, Pignata S, et al. Prospective phase II trial of trabectedin in BRCA mutated and/or BRCAness phenotype recurrent ovarian cancer patients: the MITO 15 trial. Ann Oncol 2016;27(3):487-93.

8. Kaye SB, Colombo N, Monk BJ, et al. Trabectedin plus pegylated liposomal doxorubicin in relapsed ovarian cancer delays third-line chemotherapy and prolongs the platinumfree interval. Ann Oncol 2011;22(1):49-58.

9. Ledermann J, Harter P, Gourley C, et al. Olaparib maintenance therapy in platinum-sensitive relapsed ovarian cancer. N Engl J Med 2012;366(15):1382-92. 